\title{
Transmission coordination for ad hoc networks
}

\author{
Ananth V. Kini ${ }^{\dagger}$, Steven Weber $^{\dagger}$, Fadi Aloul*, Nagarajan Kandasamy ${ }^{\dagger}$ \\ ${ }^{\dagger}$ Drexel University, Dept. of ECE, Philadelphia, PA 19104, USA \\ ${ }^{*}$ American University of Sharjah, UAE
}

\begin{abstract}
AHNs are wireless networks operating without the benefits of network infrastructure (basestations) or centralized control. AHNs working with limited spectrum perform best when simultaneous transmissions are coordinated to avoid collisions. Optimal transmission coordination is a combinatorial optimization problem that is, in general, intractable for large size networks, even with global information and central control. Constraints on simultaneous transmissions may arise from both transceiver limitations (e.g., half-duplex designs) and from requirements on the signal to interference ratio. We study the transmission coordination optimization problem under a variety of natural communication constraints. Our work identifies particular instances where the problem may be solved by greedy algorithms, and studies the performance of several natural heuristic solutions.
\end{abstract}

\section{INTRODUCTION}

Transmission coordination refers to the process of efficiently arranging simultaneous transmissions in space. This forms an important part of scheduling which involves arranging simultaneous transmissions in space, time, and frequency. We assume an ad hoc network (AHN) with limited spectrum, necessitating that concurrent transmissions must be spread out in space to avoid them generating excessive interference for their respective receivers.

Our focus in this paper is to study optimal transmission coordination under the presence or absence of various communication constraints. These communication constraints can be a result of hardware limitations of the transceiver, or the operational mode of the network. Table I gives a description of these constraints, and possible means of circumventing them.

The optimization problems seek to select a set of concurrent transmissions (edges in the communication graph) that maximize the weighted sum of the selected edges when subject to some subset of the communication constraints. Each possible subset of communication constraints is called a communication constraint set (CCS). We express each of these problems as an integer linear program (ILP). An ILP seeks to maximize a linear objective (say $\mathbf{w}^{\top} \mathbf{x}$ ) subject to linear constraints (say $\mathbf{A x} \leq \mathbf{b})$ over decision variables $\mathbf{x}=\left(x_{1}, \ldots, x_{M}\right)$ taking integer values:

$$
\max _{\mathbf{x} \in \mathbb{Z}_{+}^{M}}\left\{\mathbf{w}^{\top} \mathbf{x}: \mathbf{A x} \leq \mathbf{b}\right\}
$$

In our case each decision variable is either zero or one $\left(x_{l} \in\right.$ $\{0,1\}$ ) indicating whether or not the edge is in the selected set.

Two key concepts we will employ for solving these optimization problems are matroids and totally unimodular matrices (TUMs). Matroids are a class of subset systems (used to model constraints) for which greedy algorithms yield optimal solutions. We will classify which CCSs are matroids. TUMs
TABLE I

DESCRIPTION OF, REASONS FOR, AND MEANS TO CIRCUMVENT COMMUNICATION CONSTRAINTS.

\begin{tabular}{|l|l|l|}
\hline Constraint & Abbr. & Description \\
\hline Half-duplex & HD & $\begin{array}{l}\text { A node can not concurrently } \\
\text { transmit and receive. }\end{array}$ \\
\hline Single reception & SR & $\begin{array}{l}\text { A node can not concurrently } \\
\text { receive from multiple Tx. }\end{array}$ \\
\hline Unicast & UC & $\begin{array}{l}\text { A node can not concurrently } \\
\text { transmit distinct information } \\
\text { to multiple receivers. }\end{array}$ \\
\hline Interference & I & $\begin{array}{l}\text { A node can not receive if } \\
\text { there is any interfering } \\
\text { transmission in its vicinity. }\end{array}$ \\
\hline Constraint & $\begin{array}{l}\text { Reason for } \\
\text { constraint }\end{array}$ & $\begin{array}{l}\text { To circumvent } \\
\text { constraint }\end{array}$ \\
\hline HD & \multicolumn{3}{|l|}{ Transceiver design } & $\begin{array}{l}\text { FD transceiver, } \\
\text { multiple channels }\end{array}$ \\
\hline SR & Receiver design & Multi-user detection \\
\hline UC & $\begin{array}{l}\text { Source/channel } \\
\text { code design }\end{array}$ & $\begin{array}{l}\text { Broadcast channel } \\
\text { codes }\end{array}$ \\
\hline I & $\begin{array}{l}\text { Receiver design, } \\
\text { lack of CSI, } \\
\text { channel conditions }\end{array}$ & $\begin{array}{l}\text { Interference } \\
\text { cancellation, } \\
\text { error correction, } \\
\text { reduced data rates, } \\
\text { spread spectrum }\end{array}$ \\
\hline \multicolumn{4}{|l|}{} \\
\hline
\end{tabular}

have the property that an ILP with a TUM constraint matrix may be solved as an LP by relaxing the integrality constraint (in our case relaxing each $x_{i} \in\{0,1\}$ to each $x_{i} \in[0,1]$ ).

We will also introduce two natural heuristics and study their performance for the various problems. The length heuristic adds edges to the solution set in order of increasing/decreasing edge lengths, while satisfying the constraint criteria. The degree heuristic orders the edges by head or tail degree. If two edges have the same degree we break ties on either edge weight or edge length.

The rest of this paper is organized as follows. Section II discusses the various communication constraints, construction of the matrices for these constraints, and the formulation of the optimization problem. Related work is discussed in Section III. Section IV introduces the heuristics and the concept of unimodularity. Section ?? looks at the impact of TUM on our problem formulations. Section V investigates which problems yield a matroid structure. Section VI compares the heuristics to the optimal solution. Finally, Section VII summarizes our contributions and presents promising future directions. 


\section{COMMUNiCATION CONSTRAints AND THE OPTIMIZATION PROBLEM}

\section{A. Communication and Interference graph.}

We assume that all nodes employ unit transmission power. The communication graph $G_{c}=\left(V_{c}, E_{c}\right)$ (with $\left|V_{c}\right|=N$ and $\left.\left|E_{c}\right|=M\right)$ is directed with an edge from $i$ to $j$ indicating that $i$ is able to successfully transmit to $j$ in the absence of interference. Specifically, $\eta$ is the common noise factor at every node $v \in V_{c}$. Also, we define the symmetric $N \times N$ channel matrix $\boldsymbol{H}$ with entries $H_{u v} \in \mathbb{R}^{+}$that denote the power attenuation factor of the channel from $u$ to $v$ at a particular instant of time. A directed edge $(u, v)$ is added if the SNR from $u$ to $v\left(H_{u v} / \eta\right)$ exceeds the SNR constraint $\beta_{c}$. The interference graph $G_{i}=\left(V_{i}, E_{i}\right)$ (with $\left|V_{i}\right|=N$ ) is constructed in a similar manner with $\beta_{i}$ specifying the interference threshold, i.e., $E_{i}=\left\{(s, t): H_{s t} / \eta \geq \beta_{i}\right\}$. Both graphs $G_{i}, G_{c}$ are directed, but edges come in pairs:

$$
\begin{aligned}
& (u, v) \in E_{c}\left(E_{i}\right) \text { iff } \\
& (v, u) \in E_{c}\left(E_{i}\right)
\end{aligned}
$$

\section{B. Transmission vector.}

The point to point transmission coordination problem requires identification of an optimal subset of edges from the communication graph, $E_{S}^{*} \subseteq E_{c}$. The feasible set is exponentially large in $N$, i.e., $E_{S}^{*} \in \mathcal{P}\left(E_{c}\right)$, the power set of $E_{c}$, where $\left|\mathcal{P}\left(E_{c}\right)\right|=2^{M}$, for $M=\left|E_{c}\right|=O\left(N^{2}\right)$ the size of $G_{c}$. The transmission vector for a particular point to point schedule, say $E_{S}$, is a $\{0,1\}$-valued $M$-vector, $\mathbf{x}=\left(x_{1}, \ldots, x_{M}\right)$, with elements $x_{e}=1$ if edge $e \in E_{S}$ and $x_{e}=0$ if edge $e \notin E_{S}$. The transmission vector is the decision variable for the transmission coordination combinatorial optimization problem.

Transmission coordination schemes can be classified as maximum or maximal. A coordination scheme is maximum if its cardinality is at least as large as that of all feasible schedules. A coordination scheme is maximal if adding any additional transmissions violates one or more of the constraints. Finding the maximum coordination scheme generally requires searching over the entire state space and in some cases can be a NP-Hard problem. Maximal coordination schemes on the other hand, are easily found by greedy algorithms.

\section{Performance objective.}

The performance objective of interest is to maximize the weighted sum of the selected edges. The weight vector $\mathbf{w}=$ $\left(w_{1}, \ldots, w_{M}\right)$ has elements $w_{m} \in \mathbb{R}^{+}$denoting the relative value of activating each edge, and the transmission coordination objective is to maximize $\mathbf{w}^{\top} \mathbf{x}$. Maximizing the number of edges corresponds to $\mathbf{w}=\mathbf{1}$.

\section{Communication constraints}

The constraints are presented in linear form $(\mathbf{A x} \leq \mathbf{1})$, where $\mathbf{A}$ is $\{0,1\}$-valued and has $M$ columns. The rows of $\mathbf{A}$ indicate incompatible edges, i.e., the ones in each row in $\mathbf{A}$ represent a set (or pair) of edges that cannot be simultaneously active as they together violate one or more of the governing constraints. The number of rows in $\mathbf{A}$ varies depending on the active constraints. Here $\mathbf{1}=(1, \ldots, 1)$ is a vector of ones with the same dimension as the number of rows of $\mathbf{A}$. This mathematical formulation of the communication constraints allow us to express the point to point transmission coordination problem as a family of integer linear programming (ILP) problems, shown below. The objective is to maximize $\mathbf{w}^{\top} \mathbf{x}$ over all possible transmission vectors $\mathbf{x} \in\{0,1\}^{M}$, subject to any subset of the three primary constraints, and the interference constraint.

\begin{tabular}{rr|}
$\begin{array}{r}\text { maximize } \\
\text { over }\end{array}$ & $\mathbf{x} \in\{0,1\}^{M}$ \\
subject to any subset of & $\mathbf{w}^{\top} \mathbf{x}$ \\
(primary constraints) & $\mathbf{A}^{\mathrm{HD}} \mathbf{x} \leq \mathbf{1}$ \\
& $\mathbf{A}^{\mathrm{SR}} \leq \mathbf{1}$ \\
& $\mathbf{A}^{\mathrm{UC}} \mathbf{x} \leq \mathbf{1}$ \\
(secondary constraint) & $\mathbf{A}^{\mathrm{I}} \mathbf{x} \leq \mathbf{1}$ \\
\hline
\end{tabular}

Primary communication constraints. There are three possible primary communication constraints.

Half-duplex: Given the directed communication graph $G_{c}=\left(V_{c}, E_{c}\right)$, define $\mathcal{K}^{\mathrm{HD}}$ to be the set of head-to-tail pairs of edges

$$
\mathcal{K}^{\mathrm{HD}}=\left\{\{(i, j),(j, k)\}: i, j, k \in V_{c},(i, j),(j, k) \in E_{c}\right\} .
$$

Let $K^{\mathrm{HD}}=\left|\mathcal{K}^{\mathrm{HD}}\right|$ be the number of such edge pairs. Form the $K^{\mathrm{HD}} \times M$ matrix $\mathrm{A}^{\mathrm{HD}}$ with elements:

$$
A_{e f, g}^{\mathrm{HD}}=\left\{\begin{array}{ll}
1, & g=e \text { or } f \\
0, & \text { else }
\end{array},\right.
$$

where each row in $\mathbf{A}^{\mathrm{HD}}$ corresponds to a head-to-tail edge pair $(e, f)$ in $\mathcal{K}^{\mathrm{HD}}$. Note that each row of $\mathbf{A}^{\mathrm{HD}}$ has exactly two 1's in the positions of the two edges comprising the pair. The linear constraint $\mathbf{A}^{\mathrm{HD}} \mathbf{x} \leq \mathbf{1}$ prohibits any two edges that form a head-to-tail pair from being simultaneously active.

Single reception: a node can not concurrently receive from multiple transmitters. Define the $N \times M$ matrix $\mathbf{A}^{\mathrm{SR}}$ (with each row representing a node), and an entry:

$$
A_{j, e}^{\mathrm{SR}}=1 \text { if } e=(i, j) \text { is an incoming edge of node } j \text {. }
$$

The constraint is $\mathbf{A}^{\mathrm{SR}} \mathbf{x} \leq \mathbf{1}$, i.e., at most one incoming edge for any node can be active at a time.

Unicast: a node can not concurrently transmit distinct information to multiple receivers. Define the $N \times M$ matrix $\mathbf{A}^{\mathrm{UC}}$ (with each row representing a node), and an entry:

$$
A_{j, e}^{\mathrm{UC}}=1 \text { if } e=(i, j) \text { is an outgoing edge of node } j \text {. }
$$

The constraint is $\mathbf{A}^{\mathrm{UC}} \mathbf{x} \leq \mathbf{1}$, i.e., at most one outgoing edge of each node can be active at a time.

\section{Interference communication constraint.}

Given the directed communication graph $G_{c}=\left(V_{c}, E_{c}\right)$ and the interference graph $G_{i}=\left(V_{i}, E_{i}\right)$, define $\mathcal{K}^{\mathrm{I}}$ to be the set of pairs of edges that interfere with one another:

$$
\begin{gathered}
\mathcal{K}^{\mathrm{I}=\{}\{(i, j),(k, l)\}: i, j, k, l \in V_{c},(i, j),(k, l) \in E_{c}, \\
\left.(i, l) \in E_{i} \text { or }(k, j) \in E_{i}\right\} .
\end{gathered}
$$

Let $K^{\mathrm{I}}=\left|\mathcal{K}^{\mathrm{I}}\right|$ be the number of such edge pairs. Form the 
$K^{\mathrm{I}} \times M$ matrix $\mathbf{A}^{\mathrm{I}}$ with elements:

$$
A_{e f, g}^{\mathrm{I}}=\left\{\begin{array}{ll}
1, & g=e \text { or } f \\
0, & \text { else }
\end{array},\right.
$$

where each row in $\mathbf{A}^{\mathrm{I}}$ corresponds to a pair of conflicting edges in $\mathcal{K}^{\mathrm{I}}$. Note that each row of $\mathbf{A}^{\mathrm{I}}$ has exactly two 1 's in the positions of the two edges comprising the pair. The linear constraint $\mathbf{A}^{\mathrm{I}} \mathbf{x} \leq \mathbf{1}$ then prohibits any two edges that interfere with one another in $G_{i}$ from being simultaneously active. Refer to Fig. 1, where edges $e, f \in E_{c}$ interfere if either $(i, l) \in E_{i}$ or $(k, j) \in E_{i}$.

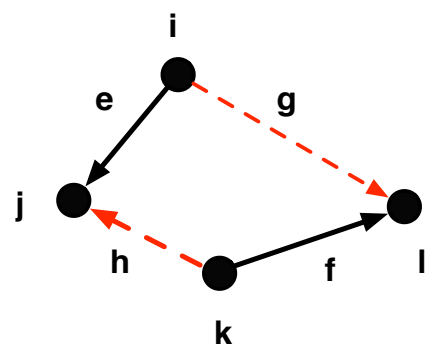

Fig. 1. Illustration of the interference (I) constraint.

\section{RELATED WORK}

Distributed throughput optimal scheduling. In their seminal paper [1], Tassiulas and Ephremides use a multi-queue system to model a multi-hop wireless network with transmissions being specified via a collection of link activation sets.

In [2] the authors show that maximum throughput algorithms based on max-weight principles yields constant-factor optimality results when the controller implements an algorithm that achieves only a constant factor of the max-weight rule every slot. In [3] the authors use a general interference model based on interference sets to show that greedy maximal scheduling achieves stability when the arrival rates are within a constant factor of the capacity region.

Recently [4] points out that the scheduling literature reveals no systematic study of the algorithmic and performance impacts of communication constraints. Performance impact refers to the effect of a CCS on the cardinality of the optimal transmission coordination scheme. In this paper the average cardinality of a maximal transmission coordination scheme as a function of the network size is studied.

Several of the optimization problems in Eqn. (3) can be related to classical problems in graph theory.

Maximum weighted matching (MWM). A matching is a set of edges not sharing a common vertex. Optimization for bidirectional point to point communication subject to all three primary constraints $(\mathrm{HD}+\mathrm{SR}+\mathrm{UC})$ is an instance of the distance-1 MWM problem. Edmonds [5] proposed an algorithm in 1965 to find a maximum matching (in both bipartite as well as non-bipartite graphs) in polynomial time. Additional related work can be found in [4].

\section{FINDING THE OPTIMAL SCHEDULE}

The problem of finding the optimum schedule is a combinatorial optimization problem. Linear programming (LP) is a technique for optimization of a linear objective function, subject to linear constraints. An ILP formulation is a LP formulation with the additional requirement that the variables can only take integer values. LP problems can be solved efficiently in most cases. ILP problems on the other hand are, in general NP-Hard. Our problem is a $0-1$ ILP or Binary Integer Programming (BIP) problem. These problems too are classified as being NP-Hard.

All of the problems in Eqn. (3) are ILPs. Relaxing the ILP to an LP (replacing $\mathbf{x} \in\{0,1\}^{M}$ with $\mathbf{x} \in[0,1]^{M}$ ) would result in the vector $\mathbf{x}$ having (in general) fractional values, which we would round up or down accordingly to yield a feasible solution to the original ILP. The optimal objective value of this LP relaxation gives an upper bound on the optimal objective value of the original ILP (because the relaxation enlarges the search space). This LP relaxation is in general not tight, i.e., we may well have non-integer solutions achieving a strictly higher objective value than any integral solution. In such a case the upper bound of the solution to the LP relaxation may be a poor estimate of the optimal solution to the original ILP. Relaxation is an appealing approach because LP problems can be solved efficiently in most cases.

Although ILP and BIP problems are classified as NP-Hard, there do exist an important subclass of problems that can be solved by LP relaxation. This arises as a result of the constraint matrix $\mathbf{A}$ and the right hand side $\mathbf{b}$ in $\mathbf{A x} \leq \mathbf{b}$ satisfying certain properties. We now review some relevant terms and theorems.

\section{A. Unimodularity}

An integer matrix of full row rank $\mathbf{A}$ is said to be totally unimodular if every square submatrix of $\mathbf{A}$ has determinant either $0,+1$, or -1 [6]. The following theorem of Hoffman and Kruskal is key to our problem.

Theorem 1 (Hoffman-Kruskal [6]): Let $\mathbf{A}$ be an $m$ by $n$ integral matrix. Then the polyhedron defined by $\mathbf{A x} \leq \mathbf{b}, \mathbf{x} \geq$ 0 is integral for every integral vector $b \in \mathbf{R}^{m}$ iff $\mathbf{A}$ is TUM.

For our optimization problems the linear constraints define a convex polyhedron called the feasible region. If a polyhedron is integral (has integer-valued vertices) the relaxation of the ILP is valid, i.e., the LP and ILP have the same solution. This is because the solution of an LP occurs at a vertex and if all vertices are integral then the solution of the LP will be feasible for the ILP. The following is another important theorem we will require.

Theorem 2 (Heller and Tompkins [7]): Let $\mathbf{A}$ be an $m$ by $n$ matrix whose rows can be partitioned into two disjoints sets $T_{1}$ and $T_{2}$, such that $\mathbf{A}, T_{1}$, and $T_{2}$ have the following properties:

- every entry in $\mathbf{A}$ is $0,+1$, or -1 ;

- every column contains at most two non-zero entries;

- if a column A contains two non-zero entries, and both have the same sign, then their rows are in different sets;

- if a column of $\mathbf{A}$ contains two non-zero entries, and they have opposite signs, their rows are in the same set.

Then $\mathbf{A}$ is TUM. 


\section{B. Heuristics}

We now introduce the various heuristics.

General heuristic.

We consider a greedy heuristic with edges sorted by several primary and secondary criteria. The general algorithm for the heuristic is shown in Algorithm (10).

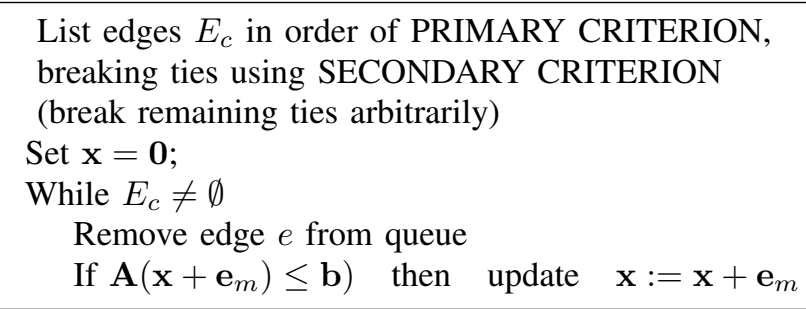

Here, $\mathbf{e}_{m}$ is the zero vector with a one in position $m$, and $\mathbf{A x} \leq \mathbf{b}$ represents the set of governing constraints.

The representative primary and secondary sorting criterion we will consider include:

1) Primary $=$ Length $(\mathrm{Inc} / \mathrm{Dec})$, Secondary $=$ none

2) Primary $=$ Degree $(\mathrm{Inc} / \mathrm{Dec})$, Secondary $=$ Length (Inc/Dec)

We will consider two special cases for edge weights: $w_{e}=1$ and $w_{e}=l_{\max } / l_{e}$, where $w_{e}$ is the weight of edge $e, l_{e}$ is the length of edge $e$, and $l_{\max }$ is the length of the longest edge. As a result of this function, the length sorting criterion is equivalent to an edge weight criterion. Also, we point out that in and out degrees are the same for all nodes, so sorting by in- and out degree yields the same ordering. For every problem we consider all the sorting criteria listed above, choosing the ones that yield the best solution.

We now demonstrate the process wherein one can check whether the LP formulations of the HD, SR, UC and I problems satisfy TUM.

1. HD constraint. Consider a network with $G_{c}$ as shown in Fig. 2. The constraint matrix $\left(\mathrm{A}^{\mathrm{HD}}\right)$ for this graph is:

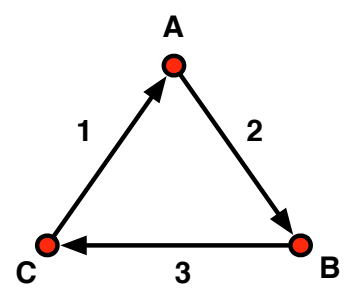

Fig. 2. Sample communication graph $G_{c}$ for a three node network.

$$
\mathbf{A}^{\mathrm{HD}}=\begin{aligned}
& 1,2 \\
& 1,3 \\
& 2,3
\end{aligned}\left(\begin{array}{lll}
1 & 2 & 3 \\
1 & 1 & 0 \\
1 & 0 & 1 \\
0 & 1 & 1
\end{array}\right)
$$

The determinant is $\left|\mathbf{A}^{\mathrm{HD}}\right|=-2 \notin\{-1,0,+1\}$. As a result the matrix is not TUM, and so the Hoffman-Kruskal theorem (Theorem 1) does not hold, meaning LP relaxation does not generate a feasible solution to the ILP.
2. SR constraint. The matrix $\mathbf{A}^{\mathrm{SR}}$ is defined in Eqn. (6). Each column corresponds to an edge, and hence each column has exactly one 1 in row $\mathrm{v}=\mathrm{h}(\mathrm{e})$. The matrix therefore is TUM by Theorem 2 (Heller and Tompkins).

3. UC constraint. The same argument holds here.

4. I constraint. The I constraint matrix has a structure similar to the HD matrix. One can show that this constraint does not satisfy TUM.

Combining a TUM problem with a non-TUM problem results in a non-TUM problem. As a result any combination of SR and UC with HD and/or I results in a non-TUM problem. Also, although not shown here one can easily prove that the SR+UC problem satisfies TUM.

\section{MATROIDS AND COMMUNICATION CONSTRAINTS}

We now explore the use of greedy algorithms in solving the transmission coordination problem. Greedy algorithms yield optimal solutions when the subset system representing the governing communications constraints is a matroid. We now proceed to define matroids and then evaluate which of the various CCSs are matroids.

\section{A. Matroids and greedy algorithms}

\section{Definition: Matroid [8]}

A matroid is an ordered pair $(\mathcal{E}, \mathcal{I})$ consisting of a finite set $\mathcal{E}$ together with a collection $\mathcal{I}$ of subsets of $\mathcal{E}$ satisfying the following three conditions:

1. $\emptyset \in \mathcal{I}$

2. If $I^{\prime} \subseteq I \in \mathcal{I}$, then $I^{\prime} \in \mathcal{I}$

3. If $I_{1}$ and $I_{2}$ are in $\mathcal{I}$ and $\left|I_{1}\right|<\left|I_{2}\right|$, then there is an element $e \in I_{2} \backslash I_{1}$ such that $I_{1} \cup\{e\} \in \mathcal{I}$

If $\mathcal{M}$ is the matroid $(\mathcal{E}, \mathcal{I})$, then $\mathcal{M}$ is called a matroid on $\mathcal{E}$. The members of $\mathcal{I}$ are called the independent sets of $\mathcal{M}$, and $\mathcal{E}$ is the ground set of $\mathcal{M}$. A subset of $\mathcal{E}$ not in $\mathcal{I}$ is called a dependent set. A minimal dependent subset $\mathcal{C}$ of $\mathcal{E}$ is called a circuit. A maximal independent set of $\mathcal{M}$ is called a base or a basis of $\mathcal{M}$. All bases of a matroid have the same cardinality. A maximal independent subset is the largest subset of $\mathcal{E}$ in $\mathcal{I}$. A minimal dependent subset is the smallest subset $E \subseteq \mathcal{E}$ s.t. $\mathcal{E} \notin \mathcal{I}$. We now look at some examples of matroids.

Matroids are related to the greedy algorithm. Under the greedy algorithm, in each step, we choose any largest weight member of $\mathcal{E}$, not already chosen, which together with the members already chosen forms a subset system while subject to some condition that maintains the property of the set $\mathcal{I}$. For any matroid $\mathcal{M}$ on $\mathcal{E}$, and for any weighting of $\mathcal{E}$, the greedy algorithm always yields the maximum weight member of the family $\mathcal{I}$ of bases of $\mathcal{M}$ [9], [10].

\section{B. Communication constraints and matroids}

We now proceed to check if the independent sets $\mathcal{I}$ for the transmission coordination problem for the various CCSs are matroids.

1. HD: Consider the simple counter-example depicted in Fig. 3. Clearly the union of any of the outgoing edges of $i$ (from $\mathcal{I}_{1}$ ) with any of the incoming edges of $i$ (from $\mathcal{I}_{2}$ ) violates the HD property. Thus this pair of subsets violates condition 3 in the matroid Definition. 
TABLE II

CATEGORIZATION OF VARIOUS PROBLEMS.

\begin{tabular}{|l|l|l|}
\hline Problem & TUM & Matroid \\
\hline HD & No & No \\
SR & Yes & Yes \\
UC & Yes & Yes \\
HD+SR & No & No \\
HD+UC & No & No \\
SR+UC & Yes & No \\
HD+SR+UC+I & No & No \\
\hline I & No & No \\
HD+I & No & No \\
SR+I & No & No \\
UC+I & No & No \\
HD+SR+I & No & No \\
HD+UC+I & No & No \\
SR+UC+I & No & No \\
HD+SR+UC+I & No & No \\
\hline
\end{tabular}

Hence the half-duplex constraint does not yield a matroid, and hence cannot be solved via use of a greedy algorithm. This should come as no surprise, since we have already established that problems involving the HD constraint cannot be solved by LP relaxation.

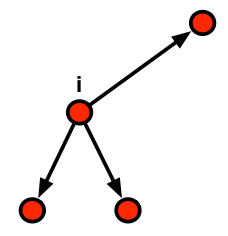

$\mathrm{II}_{1} \mathrm{I}=3$

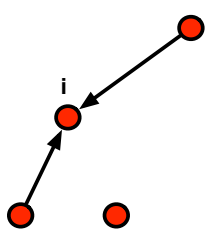

$\mathrm{II}_{2} \mathrm{I}=2$
Fig. 3. Counter-example to show that the HD constraint does not yield a matroid.

2. SR: Form the set $\mathcal{I}$ to consist of all distinct unordered subsets of edges in $\mathcal{E}$, with the following restriction: for any $i, j, k \in \mathcal{V}$, any grouping that contains edges $(i, j),(k, j)$ is an invalid grouping. This condition results in grouping of edges where each node in $\mathcal{V}$ is the head of (at most) one edge. Hence for any two groupings $\mathcal{I}_{1}, \mathcal{I}_{2} \in \mathcal{I}$, where $\left|\mathcal{I}_{1}\right|=\left|\mathcal{I}_{2}\right|+1$, we are guaranteed that $\mathcal{I}_{1}$ contains at least one edge that has as its head a node that is not the head of any edge in $\mathcal{I}_{2}$. This edge can then be added to $\mathcal{I}_{2}$ to form a grouping of cardinality $\left|\mathcal{I}_{2}\right|+1$. Hence the independent subsets $\mathcal{I}$ under the single receiver constraint form a matroid i.e., $\mathcal{M}=\left(\mathcal{E}, \mathcal{I}^{\mathrm{SR}}\right)$.

3. UC: The proof for the UC constraint is similar to the one for the SR condition above.

The SR and UC problems are the only two problem that satisfy the matroid structure property. All other problems (including the combined SR and UC problem) fail to satisfy this property. Table II summarizes these results.

\section{Importance of matroids in network design}

From a design standpoint finding the optimal transmission coordination scheme implies solving a global optimization
TABLE III

Performance (\% OF OPTIMAL SOLUtion FOR Network of $N=100$ NODES) WHEN OBJECTIVE IS TO MAXIMIZE $\mathbf{1}^{\top} \mathbf{x}$.

\begin{tabular}{|l|l|l|l|l|}
\hline Problem & $\beta_{c}=0.2$ & 0.2 & 12.0 & 12.0 \\
\hline Sorting by & length & degree & length & degree \\
\hline HD & $\mathbf{8 6}$ & 32 & $\mathbf{8 3}$ & 82 \\
SR & $\mathbf{1 0 0}$ & $\mathbf{1 0 0}$ & $\mathbf{1 0 0}$ & $\mathbf{1 0 0}$ \\
UC & $\mathbf{1 0 0}$ & $\mathbf{1 0 0}$ & $\mathbf{1 0 0}$ & $\mathbf{1 0 0}$ \\
HD+SR & 68 & $\mathbf{9 7}$ & 84 & $\mathbf{9 5}$ \\
HD+UC & 68 & $\mathbf{9 7}$ & 84 & $\mathbf{9 5}$ \\
SR+UC & 97 & $\mathbf{9 7}$ & 89 & $\mathbf{9 3}$ \\
HD+SR+UC & 97 & $\mathbf{9 8}$ & 92 & $\mathbf{9 6}$ \\
\hline I & 48 & $\mathbf{5 0}$ & 40 & $\mathbf{4 2}$ \\
HD+I & $\mathbf{7 5}$ & 51 & 35 & $\mathbf{3 9}$ \\
SR+I & 52 & $\mathbf{7 5}$ & 56 & $\mathbf{5 9}$ \\
UC+I & 61 & $\mathbf{8 8}$ & 87 & $\mathbf{9 0}$ \\
HD+SR+I & 52 & $\mathbf{7 6}$ & 35 & $\mathbf{3 9}$ \\
HD+UC+I & 60 & $\mathbf{8 7}$ & 81 & $\mathbf{9 0}$ \\
SR+UC+I & $\mathbf{8 8}$ & 81 & 87 & $\mathbf{9 0}$ \\
HD+SR+UC & 74 & $\mathbf{7 8}$ & 81 & $\mathbf{9 0}$ \\
+I & & & & \\
\hline
\end{tabular}

problem, which requires centralized control. In the best case this solution may be found in polynomial time (as in the case of the MWM problem). For most problems however, as network size increases, the explosion in search space makes centralized policies difficult and expensive. As a result one needs to make use of decentralized (distributed) policies that are able to obtain good approximations of the optimal solution in constant or polynomial times.

Matroids drastically reduce the complexity of of the optimization problem. This reduction in complexity, obviates the need of a centralized policy, making them readily scalable. This can prove to tremendously helpful when designing the network, as the designer can choose to exclude a non-matroid constraint thereby saving on the cost (complexity) associated with solving this problem.

\section{HEURISTICS VS. OPTIMAL SOLUTION}

We now compare the solutions provided by the edge length and degree heuristics to the optimal solution. We show that depending on the constraint set, either, both, or neither heuristic yields a good approximation to the optimal solution.

All edge weights, $w_{e}=1$. We evaluate the eight combinations of the primary constraints without the I constraint, and then the eight combinations with the I constraint. We present results for $\beta_{c}=0.2$ and $\beta_{c}=12.0$; this allows to us to see how the heuristics compare when the number of edges is large $\left(\beta_{c}=0.2\right)$ and small $\left(\beta_{c}=12.0\right)$. For $\beta_{c}=0.2$, and $N=100$, we set $|M|=1908$, whereas for $\beta_{c}=12.0$, and $N=100$, we set $|M|=284$.

Table III provides a summary of our results. Column 2 and 4 specify the performance when the primary sorting criterion is edge length, whereas columns 3 and 5 specify the performance when the primary sorting criterion is node degree. When the primary sorting criterion is degree, we select the secondary criterion that provides the best performance for 
each problem. Performance is expressed as a percentage of the optimal solution obtained at $N=70$.

From Table III we observe that for the $\beta_{c}=0.2$ case (without I), at least one of the heuristics yields a very good approximation of the optimal solution (with the exception of the HD case the heuristics yield an approximation that is $97 \%$ or better). For the $\beta_{c}=12.0$ case as well, we observe that at least one (and in some cases both) heuristics yield a very good approximation of the optimal solution. Observe also that for the two matroid problems, both heuristics yield the optimal solution (in fact any sorting criterion always yields the optimal solution for these problems). Upon adding the I constraint we observe that the heuristics do not perform as well. Performance of the heuristics for problems that involve the UC constraint improves (albeit marginally) when we go from $\beta_{c}=0.2$ to $\beta_{C}=12.0$, whereas the approximation for the problems that do not include this constraint worsens. We are unable to explain precisely the reason for this observation. Our intuition is that the addition of the I constraint makes the problem of finding the optimal schedule a hard one as we are trying to maximize the number of transmissions under interference. A greedy heuristic is too simplistic and ignores this constraint, resulting in a poor approximation of the optimal solution. However, when the UC constraint is in effect a node can only transmit to one other node, this reduces the number of edges that can be active, effectively allowing the heuristics to perform better. We also observe that in the absence of the I constraint, when utilizing a length sorting criterion, sorting by increasing length performs better than sorting by decreasing length. This also holds for the case where the I constraint is added to the primary constraint problems and $\beta_{i}=12.0$. However when $\beta_{c}=0.2$, we observe that in a majority of cases sorting by decreasing length performs better than sorting by increasing length. Once again we are not precisely sure as to why this occurs.

We conclude that the heuristics in general fail to achieve good performance in the presence of the I constraint.

\section{SUMMARY}

\section{A. Contributions}

To summarize:

- We characterize the various problems by way of their Primal-Dual LP formulations (we have not included this in the paper due to a lack of space).

- We prove that only the single receiver and unicast problems individually yield matroids.

- We prove that neither half-duplex nor interference problems yield matroids, nor are they TUM.

- Simulations suggest that the combined half-duplex, single receiver and unicast problem (matching problem) can be well approximated by our heuristics. This holds even with the addition of the interference problem.

- Simulations suggest that our heuristics are able to yield good approximations of the optimal solution for the problems that do not include the interference constraint.

- When operating under only the interference constraint, our heuristics fail to yield good approximations of the optimal.
- Depending on the problem at hand, either both heuristics work very well, or one outperforms the other, or neither works well.

\section{B. Future work}

There are several promising directions for future work. One such direction looks to address the problems encountered when employing LP relaxation. When employing LP relaxation to solve the original ILP, the tightness of the relaxation depends on the rounding rule employed. Ensuring that the relaxation is tight requires a certain amount of trial and error in choosing the rounding rule. An alternate approach would be to construct a feasible integral solution to the LP (Primal), while making use of a related LP (the Dual) to guide our decisions. The procedure that outlines this constitutes the Primal-Dual algorithm. This is a natural next step to try as we already have the Primal-Dual pairs for each of the problems. Along the same lines one can use semidefinite programming (SDP) techniques to solve our set of linear optimization problems. SDP is generally regarded as an extension of linear programming where the inequalities between vectors is replaced by matrix inequalities. One major difference between LP and SDP is that duality results are weaker for the latter. Nevertheless, SDP can be solved very efficiently in practice. Yet another promising direction is to look at more complex algorithms such as belief propagation techniques which have been successfully adopted in areas like iterative coding and computer vision, that involve graphs with numerous cycles.

\section{ACKNOWLEDGMENT}

The authors wish to acknowledge several helpful discussions with Dr. Ali Shokoufandeh.

\section{REFERENCES}

[1] L. Tassiulas and A. Ephremides, "Stability properties of constrained queueing systems and scheduling policies for maximum throughput in multihop radio networks," IEEE Transactions on Automatic Control, vol. 37, pp. 1936-1949, December 1992.

[2] L. Georgiadis, M. J. Neely, and L. Tassiulas, "Resource allocation and cross-layer control in wireless networks," Foundations and Trends in Networking, vol. 1, no. 1, pp. 1-144, 2006.

[3] P. Chaporkar, K. Kar, and S. Sarkar, "Throughput guarantees through maximal scheduling in wireless networks," in Proceedings of the 43rd Annual Allerton Conference on Communications, Control and Computing, Monticello, IL, November 2005, pp. 28-30.

[4] S. Weber, "Communication constraints and ad hoc scheduling," in Telecommunications Modeling, Policy, and Tehcnology, vol. 44. New York, NY, USA: Springer, 2008, pp. 275 - 289.

[5] J. Edmonds, "Paths, trees, and flowers," in Canadian Journal of Mathematics, vol. 17, 1965, pp. 449-267.

[6] A. Hoffman and J. Kruskal, "Integral boundary points of convex polyhedra," in Linear Inequalities and Related Systems; Annals of Mathematical Study (H.W. Kuhn, A.W. Tucker editions), vol. 38. Princeton, NJ, USA: Princeton University Press, 1956, pp. $223-246$.

[7] I. Heller and C. Tompkins, "An extension of a theorem of Dantzig's," in Linear Inequalities and Related Systems; Annals of Mathematical Study (H.W. Kuhn, A.W. Tucker editions), vol. 38. Princeton, NJ, USA: Princeton University Press, 1956, pp. 247 - 254.

[8] D. Welsh, Matroid Theory. Academic Press, 1976.

[9] W. Cook, W. H. Cunningham, W. R. Pullerybank, and A. Schrijver, Combinatorial Optimization. Malden, MA: Wiley-Intersciece Series in Discrete Mathematics and Optimization, 1998.

[10] T. H. Cormen, C. E. Leiserson, R. L. Rivest, and C. Stein, Introduction to Algrothims. MIT Press, 2001. 